5. Expert consensus document on management of cardiovascular diseases during pregnancy: the Task Force on the management of cardiovascular diseases during pregnancy of the European Society of Cardiology. Eur Heart J. $2003 ; 24: 761-81$.

6. Elkayam U. Pregnancy and cardiovascular disease. In: Zipes DP, Libby P, Bonow RO, Braunwald E. (editors). Braunwald's heart disease: a textbook of cardiovascular medicine. 7th ed. Philadelphia: WB Saunders; 2004. p. 1965-84.

7. Ro A, Frishman WH. Peripartum cardiomyopathy. Cardiol Rev. 2006; 14 : 35-42.

8. Pearson GD, Veille JC, Rahimtoola S, Hsia J, Oakley CM, Hosenpud JD, et al Peripartum cardiomyopathy: National Heart, Lung, and Blood Institute and Office of Rare Diseases Workshop Recommendations and Review. JAMA. 2000; 283: 1183-8.

9. Elkayam U, Akhter MW, Singh H, Khan S, Bitar F, Hameed A, et al. Pregnancy: associated cardiomyopathy. Circulation. 2005; 111: 2050-5.

10. Reimold SC, Rutherford JD. Peripartum cardiomyopathy. N Engl J Med. 2001; 344: 1629-30.

11. Sliwa K, Fett J, Elkayam U. Peripartum cardiomyopathy. Lancet. 2006; 368 : 687-93.

12. Hilfiker-Kleiner D, Kaminski K, Podewski E, Bonda T, Schaefer A, Sliwa K, et al. A cathepsin D-cleaved $16 \mathrm{kDa}$ form of prolactin mediates postpartum cardiomyopathy. Cell. 2007; 128: 589-600.

13. Lang RM, Lampert MB, Poppas A, Hameed A, Elkayam U. Peripartal cardiomyopathy. In: Elkayam U, Gleicher N. (editors). Cardiac problems in pregnancy. 3rd ed. New York: Wiley-Liss; 1998. p. 87-100.

14. Fett JD. Inflammation and virus in dilated cardiomyopathy as indicated by endomyocardial biopsy. Int J Cardiol. 2006; 112: 125-6.

15. Nabhan A. Peripartum cardiomiopathy. ASJOG. 2005; 2 (1): 231-7.

16. Kleiner DH, Meyer GP, Schieffer E, Goldmann B, Podewski E, Struman I, et al. Recovery from postpartum cardiomyopathy in two patients by blocking prolactin release with bromocriptine. J Am Coll Cardiol. 2007; 50 (24): 2354-5.

17. Elkayam U. Pregnant again after peripartum cardiomyopathy: to be or not to be? Eur Heart J. 2002; 23: 753-6.

18. Oakley C. Peripartum cardiomyopathy and other heart muscle disorders. In: Oakley C. (editor). Heart disease in pregnancy. London: Br Med J Publishing Group; 1997. p. 210-25.

19. Maron BJ, Towbin JA, Thiene G, Antzelevitch C, Corrado D, Arnett D, et al. Contemporary definitions and classification of the cardiomyopathies: AHA Scientific Statement. Circulation. 2006; 113: 1807-16.

20. Burwell and Metcalfe's: heart disease and pregnancy-physiology and management. In: Metcalfe J, McAnulty JH, Ueland K. (editors). 2nd ed. Boston: Little Brown and Company; 1986. p.133-83.

\section{Doença de Chagas}

\section{1 - Introdução}

\section{Renato Enrique Sologuren Achá}

A doença de Chagas acomete 11 milhões de pessoas nos países do Cone Sul (Argentina, Bolívia, Brasil, Chile, Paraguai e Uruguai). A prevalência da doença entre gestantes varia de $2 \%$ a $11 \%$ nos centros urbanos e de $4 \%$ a $16,4 \%$ nas áreas endêmicas ${ }^{1}$.

\section{2 - Quadro clínico}

Excluindo a fase aguda que usualmente ocorre nos primeiros anos de vida, cerca de $70 \%$ das gestantes infectadas entre 11 e 40 anos apresentam a forma indeterminada da doença, coincidindo com o período reprodutivo, e 30\% apresentam a forma crônica. A expressão "doença de Chagas sem cardiopatia aparente" refere-se às pacientes que têm sorologia positiva com eletrocardiograma e radiografia de tórax normais.

A manifestação da doença na gravidez varia desde soropositivo a alterações eletrocardiográficas, insuficiência cardíaca, arritmias, tromboembolismo e morte súbita.

Na forma indeterminada da doença, a eletrocardiografia dinâmica de 24 horas (Holter de 24 horas) comprovou que 90\% das gestantes apresentaram extrassístoles supraventriculares e 55\% extrassístoles ventriculares ${ }^{2}$. $\mathrm{Na}$ forma crônica, com miocardiopatia dilatada, pode ocorrer trombose mural endocárdica, mais frequente no átrio direito e ventrículo esquerdo, sendo causa de embolia pulmonar ou sistêmica. Nestas pacientes, cerca de $20 \%$ a $30 \%$ apresentam adelgaçamento focal do miocárdio ou aneurisma de ponta do VE, considerado patognomônico de cardiopatia chagásica. As áreas de adelgaçamento aparecem no estudo ecocardiográfico como zonas de discinesia ou acinesia ${ }^{3,4}$.

\section{3 - Diagnóstico}

1) Sorologia - Na fase aguda, exame direto e presença de anticorpos IgM anti-Trypanosoma cruzi (anti-T. cruzi) no sangue, geralmente positivo até a sexta semana da fase aguda; xenodiagnóstico; reação de Machado-Guerreiro (fixação do complemento); prova de imunofluorescência indireta para pesquisa de IgG e IgM; teste isoenzimático (Eliza);

2) ECG - Extrassístoles supraventriculares e ventriculares mono e polimórficas, taquicardia supraventricular sustentada crônica ou paroxística (flutter atrial, fibrilação atrial, taquicardias atriais, taquicardia juncional por reentrada, taquicardia A-V recíproca), taquicardia ventricular, bloqueio atrioventricular (BAV) de graus variáveis, bloqueio do ramo direito, bloqueio da divisão ântero-superior esquerda e síndromes de préexcitação, alterações da repolarização ventricular;

3) Radiografia de tórax - Realizada em casos selecionados;

4) Ecocardiograma - Fornece dados sobre o tamanho das câmaras, função miocárdica, presença de hipocinesias, acinesias ou discinesias localizadas, trombos endocárdicos;

5) Holter - Auxilia no estudo das arritmias;

6) ECG fetal - Indicado a partir da $22^{a}$ semana ${ }^{5}$.

\section{4 - Prognóstico e recomendações na gestação}

O prognóstico depende, fundamentalmente, da forma clínica da doença, do grau e tipo de arritmia e de acometimento do sistema de condução e/ou função miocárdica.

O tratamento da ICC na gestante chagásica é semelhante ao realizado fora da gestação, exceto quanto ao uso de inibidores da ECA. É recomendado o uso de hidralazina, entre 25-50 mg três vezes ao dia, e mononitrato de isossorbida, 20-40 mg duas vezes ao dia, para redução da pré e pós-carga. No caso 
de ICC refratária e com risco materno, deve ser considerada a interrupção da gestação. Nas emergências, é recomendado o uso de dobutamina na dose de $6 \mathrm{mg} / \mathrm{kg} / \mathrm{min}$.

Para arritmias venticulares, mesmo na gestação, dá-se preferência ao uso de amiodarona na dose entre 200-600 mg/ dia por via oral nos casos sintomáticos, com arritmia complexa e/ou disfunção miocárdica. O implante de marca-passo na gestação tem as mesmas indicações que fora dela, podendo ser realizado em qualquer época e sob proteção abdominal superior e inferior com avental de chumbo. A via de acesso é de preferência a região infraclavicular. A cardioversão elétrica, quando indicada, pode ser realizada em qualquer período.

\section{5 - Transmissão congênita}

A taxa de infecção congênita em recém-nascidos vivos de mães chagásicas varia de 1,6\% a 10,5\% e é certamente maior se forem considerados os abortos e os natimortos.

\subsection{1 - Mecanismo de transmissão}

A transmissão congênita da infecção chagásica é transplacentária e parece depender de fatores ligados ao parasita e ao hospedeiro. Ocorre quando os tripomastigotas presentes no espaço interviloso penetram no estroma das vilosidades, dos troncos vilosos ou da placenta corial através do epitélio trofoblástico e parasitam as células de Hofbauer (macrófagos da placenta), onde transformam em amastigotas que se multiplicam por divisão binária. Posteriormente, há transformação intracelular destas em formas tripomastigotas, que são liberadas após rotura da célula. Os tripomastigotas podem então penetrar em outros macrófagos ou atingir a luz dos vasos fetais da placenta, alcançando posteriormente o feto.

\subsection{2 - Placenta}

Apresenta reação inflamatória, com infiltração de polimorfonucleares nos espaços interviloso e viloso, com consequentes intervilosite e vilosite de intensidade variável. A placentite pode ser focal ou difusa. Quando com lesão difusa, o acometimento fetal é mais intenso, podendo levar à hidropsia e óbito fetal intrauterino. O parasitismo é frequente nessa forma, sendo raro ou mesmo ausente nas formas focais.

\subsection{3 - Avaliação laboratorial}

O exame direto do sangue periférico por meio do prévio enriquecimento (técnica de Strout) ou pela técnica de microhematócrito (creme leucocitário) é o procedimento adotado na avaliação laboratorial. O sangue deve ser colhido em 6 pequenos tubos no sentido de facilitar o encontro do T.cruzi. Ele pode ser negativo logo após o nascimento e, nestes casos, deve ser repetido (isto se deve ao fato da parasitemia aumentar gradativamente após o nascimento). Os anticorpos maternos anti-T. cruzi da fração IgG atravessam a placenta e por isso todos os recém-nascidos de mães chagásicas crônicas são soropositivos até aproximadamente o sexto mês de vida. Como os anticorpos IgM e IgA anti-T. cruzi não atravessam a placenta, a sua presença no recém-nascido indica infecção congênita. No entanto, as técnicas sorológicas dessas imunoglobulinas são de baixa sensibilidade. A mais recomendada para infecção congênita é a técnica de ELISA (IgM), por ser mais sensível $\left.\right|^{6,7,8}$.

\subsection{4 - Quadro clínico}

A doença congênita pode causar abortamento, prematuridade, retardo de crescimento intrauterino, nati e neomortalidade. A criança pode nascer com ou sem sintomas. As manifestações clínicas observadas ao nascer ou dias após o nascimento são, por ordem de frequência: hepatoesplenomegalia, febre, anemia, icterícia, edema, cianose, manifestações hemorrágicas e neurológicas (tremores, hipertonia ou convulsões), disfagia, regurgitação e desconforto respiratório.

\subsection{5 - Prognóstico}

É grave e muitos fetos morrem intraútero. Podem ocorrer manifestações digestivas precoces ou decorrentes de alterações do sistema nervoso central (meningo-encefalite, quadro clínico de paralisia cerebral).

\subsection{6 - Tratamento}

Na presença de sinais de insuficiência cardíaca fetal, com oligodrâmnios e taquicardia, o feto responde ao uso de digital e diurético por administração oral materna ou intramuscular e, em casos especiais, via cordão umbilical. A bradicardia fetal com bloqueio AV total (BAVT) congênito tem bom prognóstico; sem BAVT, o prognóstico é reservado ${ }^{9,10}$.

\subsection{7 - Aleitamento}

Nas formas aguda e crônica da doença de Chagas, o Trypanosoma cruzi pode ser isolado no leite materno. Embora possam aparecer sequelas tardias, a doença aguda no lactente tende a evoluir de forma benigna. A recomendação é que o aleitamento seja mantido em mulheres com a forma crônica da doença, exceto se houver sangramento e fissura no mamilo. Nos casos de doença aguda, a amamentação não é recomendada ${ }^{11}$.

\section{Referências}

1. Segura EL. Enfermedad de Chagas: interrupción de la transmisión en el Cone Sur de América. Rev Soc Chil Parasitol. 1995; 19: 157.

2. Achá RES, Rezende MTO, Heredia RAG, Da Silva AC, Rezende ES, Souza CAO. Prevalência das arritmias cardíacas em portadoras de doença de 
Chagas, sem cardiopatia aparente, durante e após a gravidez. Arq Bras Cardiol. 2002; 79: 1-4.

3. Born D, Achá RES, Ferraz M, Garcia M, Souza JA, Souza CAO, et al. Pregnancy and Chagas' disease. J Am Coll Cardiol. 1998; 31 (Suppl. C): 421C.

4. Herrera RN, Dias E, Pérez R, Chain S, Sant-Yacumo R, Rodriguez E, et al. Estado protrombótico en estadios tempranos en la enfermedad de Chagas crônica. Rev Esp Cardiol. 2003; 56: 377-82.

5. Pereira JB, Coura JR. Diferenças regionais na doença de Chagas: aspectos clínicos, epidemiológicos e laboratoriais de duas áreas endêmicas no Brasil. Rev Soc Bras Med Trop. 1993; 26: 70-2.

6. Bittencourt AL, Barbosa HS. Incidência de transmissão congênita da doença de Chagas em abortos. Rev Inst Med Trop Sao Paulo. 1966; 8: 184-5.

7. Bittencourt AL, Barbosa HS, Santos I, Ramos MEA. Incidência de transmissão congênita da doença de Chagas em partos a termo. Res Inst Med Trop São Paulo. 1994; 16: 197-9.

8. Bittencourt AL, Medina-Lopes MD, Camargo ME. Doença de Chagas. In: Diniz EMA, Vaz FAC. (editores). Infecções congênitas e perinatais. São Paulo: Atheneu; 1991. p. 73-89.

9. Lorca M, Veloso C, Muños P, Bahamonde MI, Garcia A. Diagnostic value of detecting specific IgA and IgM with recombinant Trypanosoma cruzi antigens in congenital Chagas' disease. Am J Trop Med Hyg. 1995; 52: 512-5.

10. Menezes CAS, Bittencourt AL, Mota E, Sherlock I, Ferreira J. Avaliação da parasitemia em mulheres portadoras de infecção pelo Trypanossoma cruzi durante e após a gestação. Rev Soc Bras Med Trop. 1992; 25: 109-13.

11. Lamounier JA, Moulin ZS, Xavier CC. Recomendações quanto à amamentação na vigência de infecção materna. J Pediatr (Rio J). 2004; 80 (5 supl.): S181-S8.

\subsection{4 - Avaliação pré-gestacional}

É a avaliação da aorta, das válvulas e da função ventricular por meio de ecocardiograma, tomografia computadorizada ou ressonância nuclear magnética. Aquelas com dilatação e disfunção ventricular devem fazer monitorização de ECG por Holter para averiguar a presença de arritmia ventricular. Como são descritos casos de morte súbita por arritmia ventricular mesmo com o uso de betabloqueadores, a possibilidade de se necessitar de um desfibrilador implantável deve ser considerada. Se constatado arco aórtico > 47 mm, recomenda-se cirurgia eletiva antes da gestação ${ }^{2}$.

\subsection{5 - Condutas na gestação ${ }^{1,2,3,4}$}

1) Pacientes com comprometimento cardíaco grave, dilatação da aorta $(>4 \mathrm{~cm}$ ) e história prévia de dissecção devem evitar a gestação. Se constatada gravidez, pode ser considerada a indicação de aborto terapêutico (IB).

2) Mesmo as pacientes consideradas de menor risco devem ter a aorta monitorada por ecocardiogramas sucessivos, preferencialmente transesofágicos, a cada 6 ou 8 semanas durante a gravidez, e de 3-6 meses após o parto. A constatação de um aumento progressivo de $10 \mathrm{~mm}$ da aorta indica cirurgia eletiva mesmo durante a gestação ou após aborto terapêutico (IC). Em revisão de pacientes submetidas à cirurgia pré-parto por dissecção da aorta tipo A, é descrita uma mortalidade materna de $15 \%$. No entanto, nos últimos anos foi observada uma redução da mortalidade materna de $30 \%$ para $0 \%$, e da fetal de $50 \%$ para $10 \%$.

3) Nos casos com indicação cirúrgica durante a gestação e com a possibilidade de viabilidade fetal (devido à alta mortalidade fetal que permanece), são recomendados parto cesáreo e cirurgia da aorta simultânea ou imediatamente após o parto (IC).

4) Atividade física vigorosa deve ser evitada e está indicado o uso de betabloqueadores durante toda gestação, sendo preferível o uso do metoprolol, por ser o mais seguro para o feto. Antes da gestação, é utilizada uma dose que mantém a FC $\leq 60$ bat./min. Se iniciado o uso durante a gestação, a dose deve ser ajustada para obter uma redução da frequência cardíaca basal em repouso $\geq 20 \%$ (IC).

5) Dissecção aguda da aorta ascendente é uma emergência cirúrgica, devendo ser realizado reparo com preservação ou 